\title{
Implementation of Infrared as a Service in Cloud-Based HNS
}

\author{
Hikaru Inomoto, Sachio Saiki, Satoshi Takatori, Seiki Tokunaga, Shinsuke Matsumoto and Masahide Nakamura \\ Graduate School of System Informatics, Kobe University \\ 1-1 Rokkodai, Nada, Kobe, Hyogo 657-8501, Japan \\ Email: inomoto@ws.cs.kobe-u.ac.jp, takatori@ws.cs.kobe-u.ac.jp, tokunaga@ws.cs.kobe-u.ac.jp, \\ sachio@carp.kobe-u.ac.jp, shinsuke@cs.kobe-u.ac.jp, masa-n@cs.kobe-u.ac.jp
}

\begin{abstract}
Home Network System (HNS) is a system which provides value-added services by orchestrating household appliances and sensors. In previous work, we have proposed Cloud HNS, which is a new type of HNS based on the concept of Cloud. In this research, we propose IRaaS (Infrared as a Service) that realizes networking control of household appliances controlled by infrared signal, as a Cloud HNS's appliance control service. IRaaS consists of two components, IR Proxy and jRemocon. IR Proxy mediates appliance control on Cloud, and jRemocon transmits an infrared signal in a home. With these components, IRaaS can provide appliance control functions as a service on Cloud. Then, we design and consider elements required for implementing IRaaS, and we also create and test a prototype. Based on these results, we confirm the sufficiency of functions of IRaaS.
\end{abstract}

Keywords-Cloud-based HNS, Appliance control, IRaaS

\section{INTRODUCTION}

\section{A. Background}

Home Network System (HNS) is an emerging ubiquitous system in today's advanced information society [1]. HNS provides various value-added services by orchestrating networked home appliances and sensors. Some examples of HNS services are: energy-saving service [2], appliance control service [3] and context aware service [4]. Recently, some applications using HNS have appeared on the market.

In spite of long-term research and development, HNS have not become common in our society. One of the major reasons is that HNS appliances and services are tightly coupled together. This is due to a business model, which is generally called "vendor lock-in". Because of the coupling, it is difficult to provide multi-vendor HNS services on a single HNS environment. The other reasons include high cost of installing, or psychological barrier from reliability. In general, HNS uses household appliances that controlled over network, and HNS can not use legacy appliances, which is not available network controlling. Therefore people must renew their home appliances to the compatible ones for installing HNS, and this leads high cost of installing it. HNS user's daily life deeply depends on HNS, therefore the system must avoid being failure. However, a home server, which plays a central role of HNS, exists in the user's home in the conventional HNS system. An internal home server may become a single point of failure in HNS system and makes psychological uncertainty.

\section{B. Cloud HNS}

In order to solve these problems, we have proposed a new HNS architecture based on the concept of Cloud, named Cloud HNS [5]. In Cloud HNS, all functions of home server are delegated to Cloud and provided as Web services. Home server exists not in a home but on Cloud, this leads to reducing psychological barrier with maintenance. Abstraction of functions leads to realization of services regardless of appliance vendor.

Cloud HNS uses many web services for performing various functions, for instance, appliance control, getting sensor information. To use these services, Cloud HNS resolves which services the objective needs, and what parameters the services require. Cloud HNS manages this information in databases on Cloud, and resolves the information using the databases. Moreover, the databases include appliance composition of household or which controller terminals there are in user's rooms.

\section{Objective and method}

In Cloud HNS, Appliance control methods are also abstracted, Cloud HNS performs appliance control using services that mediates between the appliances and network. Using these services, Cloud HNS can perform controlling legacy appliances. Thus, people can use their traditional home appliances in HNS without purchasing networked appliances.

In general, infrared signal is widely popular as appliance control method, however, HNS can not use the appliances controlled by an infrared signal. In this research, we propose IRaaS (Infrared as a Service) that can realize controlling of the appliances over network, as a Cloud HNS's appliance control service.

IRaaS consists of two components, IR Proxy and jRemocon. IR Proxy is a service that delegates control request to $\mathrm{jRemocon}$, and $\mathrm{jRemocon}$ is a device that transmits an infrared signal to execute appliance control. With these components, IRaaS can execute requested appliance control, and HNS user can use their legacy appliances on HNS without replacing them.

In this paper, we design and consider elements required for implementing IRaaS. The elements specifically include the API of IRaaS, how IR Proxy manages infrared signals, and signal emission board jRemocon needs. We also create and test a prototype of IRaaS, and as a result, we confirm the sufficiency of functions of IRaaS in a practical environment. 


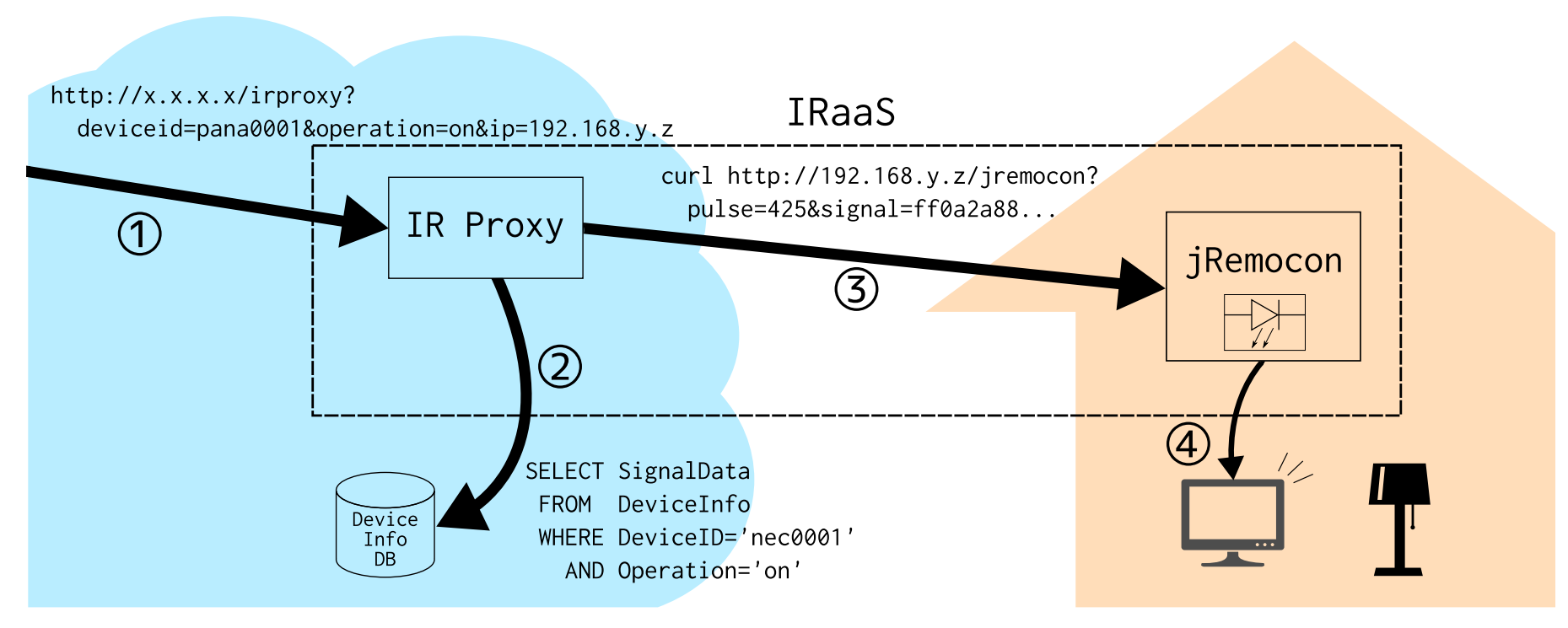

Fig. 1. Architecture of IRaaS

\section{IRAAS : INFRARED AS A SERVICE}

IRaaS represents a Cloud HNS service which controls home appliances with infrared interface. In this section, details of IRaaS are described as follows, and to confirm the sufficiency of functions of IRaaS. Then, we build a prototype and check under real environment.

\section{A. Architecture of IRaaS}

IRaaS consists of two components, IR Proxy and jRemocon. IR Proxy is a service on Cloud, which sends a necessary data for a target appliance to jRemocon when IR Proxy receives an appliance control request. jRemocon is a device installed in a house, which transmits an infrared signal corresponding to received data.

Infrared signal to control appliance is represented by a set of pulse-width and bit stream. A text encoded from the set is defined as Signal Data, and which IR Proxy send to jRemocon.

An overall architecture of IRaaS is shown as Fig. 1. Each arrow indicates its operations. In the following, a series of execution of the appliance control is shown.

1) Identifying a home-unique information needed to requested appliance control, Cloud HNS delegates the request to IR Proxy.

2) Getting the request, IR Proxy resolves a necessary Signal Data to perform the request.

3) IR Proxy sends the Signal Data to jRemocon.

4) jRemocon transmits the Signal Data as an infrared signal.

As shown above, IRaaS realizes appliance control by combining the functions of IRaaS's components, which are Signal Data resolving function of IR Proxy and infrared signal transmission function of $\mathrm{jRemocon}$.

All of Cloud HNS's functions must be implemented as referable services. To accommodate the request from Cloud
HNS, the components of IRaaS, IR Proxy and jRemocon have been implemented WebAPI. As a concrete method, these components are deployed on HTTP Server as CGI programs.

\section{B. IR Proxy}

The main functions of IR Proxy are resolving and sending Signal Data. IR Proxy's API arguments are DeviceID, Operation, ControllerAddress. DeviceID and Operation specify an operation user wants, and ControllerAddress specifies a jRemocon which transmits a signal. Receiving an appliance control request, IR Proxy resolves a Signal Data corresponding to input. To resolve a Signal Data, IR Proxy uses a database named Device Information Database. The database has records that have DeviceID, Operation, and Signal Data corresponding to them. IR Proxy resolves the Signal Data by searching the database, then IR Proxy sends the data to a jRemocon identified by ControllerAddress.

At a single operation of a single appliance, corresponding infrared signal is unique regardless of a house where the appliance is installed in. Therefore, HNS users need no their own database by sharing a master database that has Signal Data corresponding all appliance operations. To implement it, Device Information Database exists on Cloud, and is shared by IR Proxies used by all houses. In Cloud HNS, the Signal Datas in the database are provided by household appliance vendors.

With this prototype, MySQL as Device Information Database and Apache HTTP Server as CGI platform are installed on a virtual machine created on Cloud server. Receiving an appliance control request via WebAPI, CGI program resolves a corresponding Signal Data by querying the database with MySQL command. The query is shown in Fig. 1 with arrow 2, and constructed to get a record that matches inputted DeviceID and Operation. The resolved Signal Data is sent to a jRemocon identified by ControllerAddress. In the prototype system, curl command is used for sending data. 


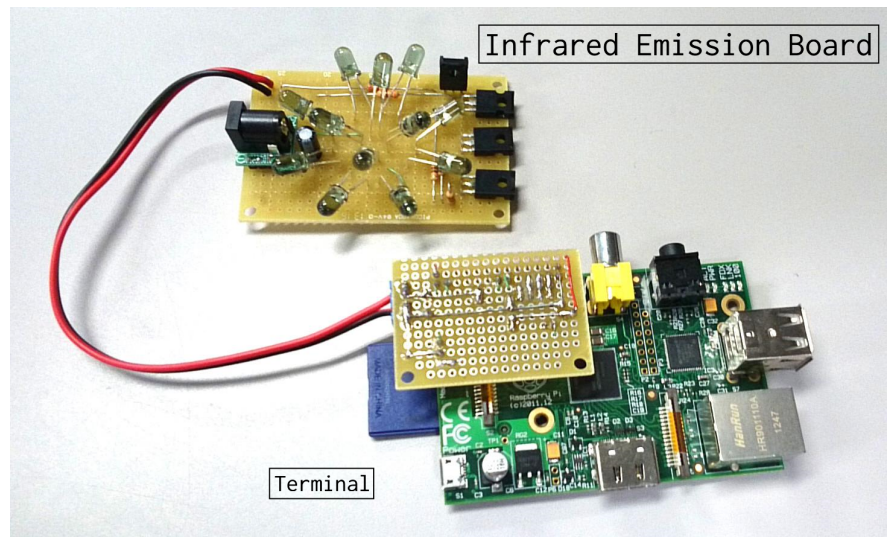

Fig. 2. A prototype of $\mathrm{j}$ Remocon

\section{C. jRemocon}

The main function of $\mathrm{jRemocon}$ is transmitting an infrared signal. jRemocon receives Signal Data from IR Proxy via own WebAPI, and converts a Signal Data to an infrared signal. The feature of jRemocon is only transmitting a Signal Data as an infrared signal. All of other tasks are performed on Cloud. With this architecture design, the terminal installed in a home only have a simple device. Therefore, the terminal can be exchanged with lower cost if it becomes out of order. Furthermore, the implementation of IRaaS about all infrared signal function is realized in software. This implementation leads to possibility for representing various infrared signals formats even if the format is a special case. That also leads to enabling an alternative support for new appliances which uses unknown format with only software modification.

Given a usage of jRemocon in a home, the terminal has to become as small as possible. Therefore, we implement jRemocon using Raspberry $\mathrm{Pi}$, which is a tiny computer having digital IO pins. For transmitting signals, I create an infrared light emission circuit board, called Infrared Emission Board. On the terminal, LIRC, which is a software package that decodes and sends infrared signal, and Apache as CGI platform are installed. Fig. 2 shows a prototype of jRemocon. As shown in the figure, a Raspberry $\mathrm{Pi}$ as a terminal and an Infrared Emission Board are connected. To control a particular appliance, jRemocon must be able to transmit accurate infrared signal conforming to the low-level signal format. By using LIRC, transmitting of promised infrared signal is assured.

After Receiving a Signal Data via WebAPI, an internal CGI program of $\mathrm{jRemocon}$ converts the Signal Data into the LIRC-compatible signal. LIRC transmits the infrared signal based on the converted signal through by the Infrared Emission Board. Infrared Emission Board has components for infrared light emission, e.g. infrared LED or transistor. The circuit board blinks infrared light according to Raspberry pi's IO pin controlled by the LIRC. For household appliance controller, it is necessary to provide the adequate quantity of light and wide range emission. Most of infrared LED has high directivity, therefore we apply the LED array method which attaches multiple of LEDs in radial fashion in order to realize widely signal emission. Furthermore, constant current circuit can supply stable current to all LEDs, thus this makes all LEDs emit with enough luminance.

\section{Operation trial}

To confirm the sufficiency of functions of IRaaS, we have conducted some appliance control trial in our laboratory room. The dimensions of the room have about $10[\mathrm{~m}] \times 8[\mathrm{~m}]$. In most of case, this is large enough as a general house. As a conventional appliance control device in the room, a networkconnected learning remote controller has been used. For a user interface of the controller, web browser application also has been used. In the trial, some appliance operations (e.g. turning tv or light on/off) are performed on the application that is modified to use IRaaS as an appliance controller.

The results of the trial are summarized as follows. We can confirm that IRaaS has enough functions and performance for the practical conditions. At least, appliance control features are equivalent to the traditional learning remote controller.

\section{CONCLUSION}

In this paper, we proposed a novel Cloud service named IRaaS which controls legacy appliances on Cloud HNS. IRaaS can perform controlling legacy appliances, by mediating between infrared signal and network. By using IRaaS, Cloud HNS users can control IR based appliance via Cloud service. The sufficiency of features and performance are confirmed through a practical trial.

Future works include implementation jRemocon with smaller, lightweight hardware and considering in networkdisabled environment. These points should be investigated further.

\section{ACKNOWLEDGMENT}

This research was partially supported by the Japan Ministry of Education, Science, Sports, and Culture [Grant-inAid for Scientific Research (C) (No.24500079, No.24500258), Scientific Research (B) (No.26280115), Young Scientists (B) (No.26730155)] and Kawanishi Memorial ShinMaywa Education Foundation.

\section{REFERENCES}

[1] M. Nakamura, A. Tanaka, H. Igaki, H. Tamada, and K. Matsumoto, "Constructing home network systems and integrated services using legacy home appliances and web services," International Journal of Web Services Research, vol. 5, no. 1, pp. 82-98, Jan 2008.

[2] M. A. A. Pedrasa, T. D. Spooner, and I. F. MacGill, "Coordinated scheduling of residential distributed energy resources to optimize smart home energy services,' IEEE Transactions on Smart Grid, vol. 1, no. 2, pp. 134-143, Sept 2010.

[3] Y. Tajika, T. Saito, K. Teramoto, N. Oosaka, and M. Isshiki, "Networked home appliance system using bluetooth technology integrating appliance control/monitoring with internet service," IEEE Transactions on Consumer Electronics, vol. 49, no. 4, pp. 1043-1048, Nov 2003.

[4] Y. S. Chen, I. C. Chen, and W. H. Chang, "Context-aware services based on osgi for smart homes," in Ubi-media Computing (U-Media), $20103 \mathrm{rd}$ IEEE International Conference on, July 2010, pp. 38-43.

[5] S. Takatori, S. Tokunaga, J. Lee, S. Matsumoto, and M. Nakamura, "A cloud-based architecture for home network system," in IEEE International Workshop on Cloud-integrated Cyber Physical Systems 2014, vol. 113, December 2014, pp. 268-270. 